\title{
MALAT1 participates in ultraviolet B-induced photo-aging via regulation of the ERK/MAPK signaling pathway
}

\author{
LI LEI $^{1-3 *}$, QINGHAI ZENG ${ }^{1 *}$, JIANYUN LU $^{1}$, SHU DING $^{1}$, FANG XIA $^{4}$, JIAN KANG $^{1}$, LINA TAN $^{1}$,

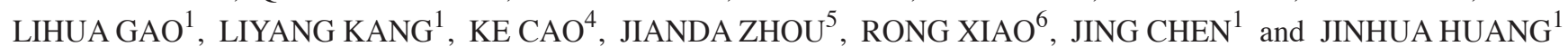 \\ ${ }^{1}$ Department of Dermatology, Third Xiangya Hospital of Central South University, Changsha, Hunan 410013; \\ Departments of ${ }^{2}$ Dermatology and ${ }^{3}$ Hunan Key Laboratory of Skin Cancer and Psoriasis, Xiangya Hospital, \\ Central South University, Changsha, Hunan 410008; Departments of ${ }^{4}$ Oncology and ${ }^{5}$ Plastic and Reconstructive \\ Surgery, Third Xiangya Hospital of Central South University, Changsha, Hunan 410013; ${ }^{6}$ Department of \\ Dermatology, Second Xiangya Hospital, Central South University, Changsha, Hunan 410011, P.R. China
}

Received January 16, 2016; Accepted February 2, 2017

DOI: $10.3892 / \mathrm{mmr} .2017 .6532$

\begin{abstract}
Long non-coding RNA (lncRNA), transcripts of $>200$ bp in length that do not appear to exhibit any coding capacity, are important in the occurrence and development of cancer, cardiovascular and neurological diseases. However, effects of lncRNAs on photo-aging remain to be elucidated. To explore the potential effects of the lncRNA metastasis-associated lung adenocarcinoma transcript 1 (MALAT1) on photo-aging in fibroblasts, MALAT1 expression was silenced in fibroblasts using small interference RNA. Reverse transcription-quantitative polymerase chain reaction (RT-qPCR) was used to examine MALAT1 expression in normal and silenced fibroblasts following irradiation with $60 \mathrm{~mJ} / \mathrm{cm}^{2}$ ultraviolet B (UVB) and an ELISA assay was used to identify matrix metalloproteinase-1 (MMP-1) content in the cellular supernatant. A $\beta$-galactosidase kit was applied to measure the number of senescent cells and a western blot assay
\end{abstract}

Correspondence to: Professor Jinhua Huang or Professor Jing Chen, Department of Dermatology, Third Xiangya Hospital of Central South University, 138 Tongzipo Road, Changsha, Hunan 410013, P.R. China

E-mail: huangjinhua60@163.com

E-mail: 43700351@qq.com

*Contributed equally

Abbreviations: DCFH-DA, dichloro-dihydro-fluorescein diacetate; DMEM, Dulbecco's modified Eagle's medium; ERK, extracellular signal-regulated kinase; JNK, c-Jun N-terminal kinase; lncRNA, long non-coding RNA; MALAT1, metastasis-associated lung adenocarcinoma transcript 1; MMP-1, metalloproteinase-1; MAPK, mitogen-activated protein kinase; NAC, N-acetyl-L-cysteine; PBS, phosphate-buffered saline; P-ERK, phosphorylated ERK; P-JNK, phosphorylated JNK; P-p38, phosphorylated p38; ROS, reactive oxygen species; siRNA, small interference RNA; UVB, ultraviolet B

Key words: long non-coding RNAs, metastasis-associated lung adenocarcinoma transcript 1, photo-aging, matrix metalloproteinase1 , mitogen-activated protein kinase, reactive oxygen species was used to detect extracellular signal-regulated kinase (ERK), c-Jun N-terminal kinase (JNK) and p38 phosphorylation levels. RT-qPCR was additionally used to detect changes in MALAT1 expression following suppression of UVB-induced reactive oxygen species (ROS) generation with $\mathrm{N}$-acetyl-L-cysteine (NAC). Fibroblasts irradiated with $60 \mathrm{~mJ} / \mathrm{cm}^{2} \mathrm{UVB}$ demonstrated increased MALAT1 expression, MMP-1 secretory volume and number of senescent cells, and greater levels of ERK, p38 and JNK phosphorylation. Following silencing of MALAT1 expression in photo-aged fibroblasts, decreases were observed in MMP-1 secretory volume, number of senescent cells and phosphorylation levels of ERK. NAC reduced ROS content, however, it did not affect MALAT1 expression. Therefore, it was concluded that MALAT1 may participate in UVB-induced photo-aging via regulation of the ERK/mitogen-activated protein kinase signaling pathway and UVB-induced MALAT1 expression is independent of ROS generation.

\section{Introduction}

Photo-aging of skin primarily refers to ultraviolet (UV) irradiation-induced skin aging, which generally manifests as exposed skin laxity, sagging, wrinkles, pigmentation, dryness, roughness, telangiectasia and the appearance of large pores (1). Primary histological features of photo-aged skin include reduction of collagen and accumulation of abnormal elastic fibers (2). UV irradiation may induce abundant secretion of matrix metalloproteinases (MMPs) from fibroblasts and keratinocytes, particularly matrix metalloproteinase-1 (MMP-1), which results in abnormal degradation of the extracellular matrix (1). MMP-1 expression during photo-aging is primarily controlled by the mitogen-activated protein kinase (MAPK) signaling pathway (3). A previous study confirmed that UV-induced reactive oxygen species (ROS) promote MMP-1 expression and induce the occurrence of photo-aging by upregulating phosphorylation levels of the MAPK signaling pathway elements c-Jun N-terminal kinase (JNK), extracellular signal-regulated kinase (ERK) and p38 (4).

Long non-coding RNA (lncRNA) transcripts that are $>200$ bp in length, which do not appear to exhibit 
coding capacity, regulate gene expression at transcriptional, post-transcriptional and translational levels $(5,6)$. Metastasis-associated lung adenocarcinoma transcript 1 (MALAT1), an evolutionarily conserved lncRNA expressed in a wide range of species and tissues, exhibits biological functions including nuclear organization and architecture, gene splicing, and epigenetic modulation of gene expression $(7,8)$. MALAT1 is important in the occurrence and development of cancer, cardiovascular and diabetes-associated microvascular disease (9-11). It has previously been demonstrated that MALAT1 interacts with the MAPK signaling pathway. MALAT1 may activate ERK/MAPK signaling to promote proliferation and metastasis of gallbladder cancer cells (12). Following silencing MALAT1 expression, the p38/MAPK signaling pathway has been demonstrated to be downregulated in endothelial cells (10). Activation of MAPK signaling exerts a key effect on photo-aging, and the present study aimed to investigate the role of MALAT1 in UV-induced photo-aging and any potential interaction with the MAPK signaling pathway elements.

The present study is the first, to the best of the authors' knowledge, to demonstrate that $60 \mathrm{~mJ} / \mathrm{cm}^{2} \mathrm{UVB}$ irradiation induces high expression of MALAT1 in fibroblasts. MALAT1 siRNA potentially suppressed UVB-induced MMP-1 secretion and fibroblast senescence by inhibiting activation of the ERK/MAPK signaling pathway.

\section{Materials and methods}

Primary culture offibroblasts. Foreskin tissue was donated (with signed informed consent) by a healthy young male following circumcision. The donor's health status was established through physical examinations and laboratory investigations which revealed no obvious abnormalities. The sample was immersed in an iodine complex for $15 \mathrm{~min}$ and washed three times with phosphate buffered saline (PBS). Following removal of subcutaneous tissue, the sample was cut into pieces and digested with trypsin to isolate fibroblasts. Fibroblasts were collected, washed, and then incubated with high-glucose Dulbecco's Modified Eagle's medium (DMEM; Gibco; Thermo Fisher Scientific, Inc., Waltham, MA, USA) supplemented with $10 \%$ fetal bovine serum (HyClone; GE Healthcare Life Sciences, Logan, UT, USA). Cells in logarithmic phase from the third to eighth passage were used for experiments.

Ultraviolet B (UVB) irradiation. Cells that had reached $\sim 70 \%$ confluence were irradiated with UVB using a SS-07 light therapy device $(36 \mathrm{~W}$ power; time of irradiation automatically adjusted to dose of irradiation; cat. no. 1,047,469; Shanghai Sigma Hi-Tech Co., Ltd., Shanghai, China). Prior to irradiation, culture medium was removed from cells, which were then washed twice with PBS. Fibroblasts, coated with a thin layer of PBS, were vertically irradiated with UVB immediately following opening the culture dish cover. The distance of irradiation was $20 \mathrm{~cm}$. Complete medium was added to the culture dish immediately following irradiation and the culture dish was then incubated in the original culture environment.

Chemical treatments. MALAT1 siRNA (5'-CACAGGGAA AGCGAGTGGTTGGTAA-3') and the negative control non-targeting siRNA were designed and synthesized by GeneChem Co., Ltd. (Shanghai, China). When cells reached 30-50\% confluence, siRNAs (50 nM) were transfected into cells using Lipofectamine ${ }^{\circledR} 3000$ (Thermo Fisher Scientific, Inc.,) in serum-free DMEM according to the manufacturer's protocol. Following $6 \mathrm{~h}$ of incubation, cells were returned to normal medium in the incubator. When cells reached $70 \%$ confluence, they were exposed to irradiation. Cells were pretreated with $10 \mathrm{mM}$ N-acetyl-L-cysteine (NAC; Beyotime Institute of Biotechnology, Haimen, China) $1 \mathrm{~h}$ prior to irradiation. Following this, cells were supplemented with 10 mM NAC for $24 \mathrm{~h}$.

Cell proliferation. Cells were seeded into a 96-well plate $\left(10^{4}\right.$ cells/well). Confluent cells were treated with serum-free medium for $24 \mathrm{~h}$ and irradiated with UVB. Following irradiation, cells were incubated with normal medium for $24 \mathrm{~h}$ and then treated with $10 \mu \mathrm{l}$ Cell Counting kit (CCK)-8 (Dojindo Molecular Technologies, Inc., Kumamoto, Japan) for $4 \mathrm{~h}$. Absorbance values were measured at a wavelength of $450 \mathrm{~nm}$ using a microplate reader (GEN5 CHS v.2.00, BioTek Instruments, Inc., Winooski, VT, USA).

$R T$ - $q P C R$. Total RNA was extracted from cells using TRIzol ${ }^{\circledR}$ reagent (Thermo Fisher Scientific, Inc.). Extracted total RNA, following purification and quality assessments, was reverse-transcribed to cDNA. RT-qPCR was performed to detect MALAT1 expression using the SYBR-Green PCR Master Mix (Applied Biosystems; Thermo Fisher Scientific, Inc.) with a ABI PRISM 7700 system (Applied Biosystems; Thermo Fisher Scientific, Inc.). The thermocycling conditions were as follows: Initial denaturation at $95^{\circ} \mathrm{C}$ for $2 \mathrm{~min}$, then denaturation at $95^{\circ} \mathrm{C}$ for $15 \mathrm{sec}$, annealing at $56^{\circ} \mathrm{C}$ for $15 \mathrm{sec}$ and extension at $68^{\circ} \mathrm{C}$ for $20 \mathrm{sec}$, for a total of 40 cycles. $\beta$-actin served as an internal reference. The expression level of MALAT1 was calculated using the $2^{-\Delta \Delta \mathrm{Cq}}$ method (13) method. The primer sequences used were as follows: Forward, 5'-AAAGCA AGGTCTCCCCACAAG-3' and reverse, 5'-GGTCTGTGCTAGATCAAAAGGCA-3' for MALAT1. Experiments were performed in triplicate.

Enzyme-linked immunosorbent assay (ELISA) for MMP-1 expression in supernatants. Following irradiation, cells were incubated with serum-free medium for $24 \mathrm{~h}$. Cell culture medium was collected and centrifuged at $1,000 \mathrm{x} \mathrm{g} / \mathrm{min}$ for $10 \mathrm{~min}$ at room temperature, and then supernatants were harvested. MMP-1 concentration was detected in supernatants using the MMP-1 kit (cat. no. EK0458; Boster Systems, Inc., Pleasanton, CA, USA) according to the manufacturer's protocol.

Detection of senescent cells using a $\beta$-galactosidase (SA- $\beta$-Gal) kit. Following 24 h of UVB irradiation, cells were stained using an SA- $\beta$-Gal staining kit (Beyotime Institute of Biotechnology) according to the manufacturer's protocol. Senescent cells presented with a blue color around the nuclei and the percentage of senescent cells was equal to number of cells stained blue/total number of cells x $100 \%$.

Detection of ROS content using flow cytometry. Following $24 \mathrm{~h}$ of UVB irradiation, cells were treated with serum-free 

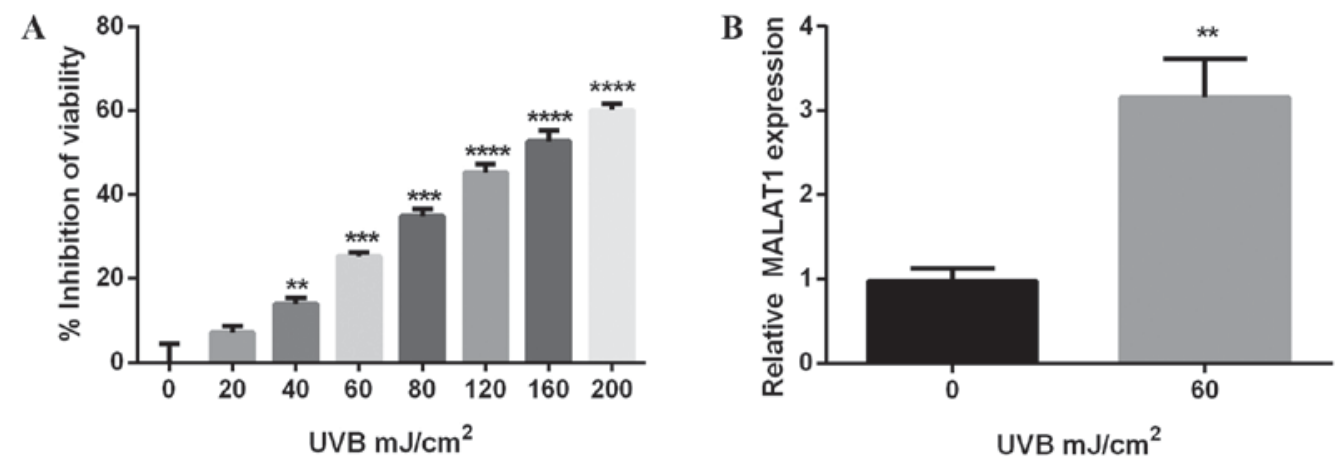

Figure 1. A total of $60 \mathrm{~mJ} / \mathrm{cm}^{2} \mathrm{UVB}$ upregulates MALAT1 expression in fibroblasts. (A) Effect of UVB irradiation on cell viability, detected via Cell Counting kit- 8 . The inhibitory rate on cell viability increased in a dose-dependent manner. The inhibitory rate was approximately $25 \% \mathrm{in}$ the $60 \mathrm{~mJ} / \mathrm{cm}^{2}$ group. (B) Reverse transcription-quantitative polymerase chain reaction demonstrated an increase in MALAT1 expression with $60 \mathrm{~mJ} / \mathrm{cm}^{2} \mathrm{UVB}$. ${ }^{* * * *} \mathrm{P}<0.0001$, ${ }_{* * *} \mathrm{P}<0.001$ and ${ }^{* *} \mathrm{P}<0.01$ vs. control. UVB, ultraviolet B; MALAT1, metastasis-associated lung adenocarcinoma transcript 1.

medium containing $10 \mu \mathrm{M}$ dichloro-dihydro-fluorescein diacetate (DCFH-DA; cat. no. KGT010-1; Nanjing Keygen Biotech Co.,Ltd., Nanjing, China) for $30 \mathrm{~min}$ at $37^{\circ} \mathrm{C}$, according to the manufacturer's protocol, and then washed three times with DMEM. Images were captured using a fluorescence microscope. Cells were collected and the level of green fluorescence was detected to evaluate the levels of intracellular ROS using flow cytometry (FACSCalibur, BD Biosciences, Franklin Lakes, NJ, USA) with BD CellQuest Pro software version 6.0 (BD Biosciences) according to the manufacturer's protocol.

Western blot analysis. Following fibroblast irradiation with UVB for $48 \mathrm{~h}$, total protein was collected. Expression levels of ERK, phosphorylated ERK (p-ERK), p38, phosphorylated p38 (p-p38), JNK and phosphorylated JNK (p-JNK) were measured using western blot analysis. The process was conducted as previously described (14). Briefly, the cells were rinsed with PBS and lysed in radioimmunoprecipitation lysis buffer (Beyotime Institute of Biotechnology) with a protease and phosphatase inhibitor cocktail (Thermo Fisher Scientific, Inc.) on ice for $30 \mathrm{~min}$. Cell lysates were centrifuged at $13,000 \times \mathrm{g}$ at $4^{\circ} \mathrm{C}$ for $20 \mathrm{~min}$. The supernatants were collected and the proteins were quantified using a bicinchoninic acid protein assay kit (Beyotime Institute of Biotechnology). Protein samples $(20 \mu \mathrm{g})$ were separated by $10 \%$ sodium dodecyl sulfate-polyacrylamide gel electrophoresis. Gels were transferred to nitrocellulose membranes, blocked with 5\% non-fat-milk and then probed with anti-ERK (dilution, 1:1,000; Cell Signaling Technology, Inc., Danvers, MA, USA; cat. no. 9926), anti-p-ERK (dilution, 1:1,000; Cell Signaling Technology, Inc.; cat. no. 9910), anti-p38 (dilution, 1:1,000; Cell Signaling Technology, Inc.; cat. no. 9926), anti-p-p38 (dilution, 1:1000; Cell Signaling Technology, Inc.; cat. no. 9910), anti-JNK (dilution, 1:1,000; Cell Signaling Technology, Inc.; cat. no. 9926), or anti-p-JNK (dilution, 1:1,000; Cell Signaling Technology, Inc.; cat. no. 9910) antibody at $4^{\circ} \mathrm{C}$ overnight. Following incubation with a rabbit horseradish peroxidase-conjugated secondary antibody (dilution, 1:2,000; Abcam, Cambridge, UK; cat. no. ab6721) at room temperature for $2 \mathrm{~h}$, immunoreactive bands were visualized using a electrochemiluminescence detection reagent (Yeasen; Shanghai Yi Sheng Biological Technology Co., Ltd., Shanghai, China; cat. no. 36208ES60) according to the manufacturer's instructions. Integrated optical density (IOD) was calculated using ImageJ version 1.46 software (http://rsb.info.nih.gov/ij). The relative intensity of the level of the protein of interest was calculated using the following formula: $\mathrm{IOD}_{\text {phosphorylated protein }}$ band of interest $/ \mathrm{IOD}_{\text {corresponding total protein band }}$. Representative blots of at least three independent experiments are presented.

Statistical analysis. Data were analyzed using SPSS software, version 22.0 (IBM SPSS, Armonk, NY, USA) and expressed as the mean \pm standard deviation. All experiments were performed at least in triplicate. Significance tests were conducted on the data groups using analysis of variance followed by a comparison between the specific groups using a Student-Newman-Keuls test and analysis of differences between only two groups was performed using an unpaired Student's t-test. $\mathrm{P}<0.05$ was considered to indicate a statistically significant difference.

\section{Results}

MALAT1 expression increases following UVB irradiation. To investigate the phototoxicity of UVB on fibroblasts, cell viability was detected using the CCK- 8 method, on cells irradiated with various doses of UVB for $24 \mathrm{~h}$. The results demonstrated UVB suppressed melanocyte viability, an inhibitory effect enhanced by increasing doses of UVB. The inhibitory rate of $60 \mathrm{~mJ} / \mathrm{cm}^{2} \mathrm{UVB}$ was $\sim 25 \%(\mathrm{P}<0.001$; Fig. 1A) and results further demonstrated that $60 \mathrm{~mJ} / \mathrm{cm}^{2} \mathrm{UVB}$ increased MALAT1 expression ( $\mathrm{P}<0.01$; Fig. 1B).

MALAT1 siRNA inhibits UVB-induced MMP-1 secretion. To investigate the effects of MALAT1 on photo-aging, MALAT1 expression in fibroblasts was silenced and effects on MMP-1 secretion observed. The results demonstrated that MALAT1 siRNA suppressed MALAT1 expression $(\mathrm{P}<0.01$ and $\mathrm{P}<0.0001$; Fig. 2A) and inhibited $60 \mathrm{~mJ} / \mathrm{cm}^{2}$ UVB-induced MMP-1 secretion ( $\mathrm{P}<0.01$ and $\mathrm{P}<0.001$; Fig. $2 \mathrm{~B})$.

MALAT1 siRNA inhibits UVB-induced fibroblast senescence. The ratio of senescent cells was markedly greater following UVB irradiation (74.4\%) compared with cells that had not been exposed to irradiation (15.7\%). In addition, the ratio of senescent cells was significantly lower following intervention 

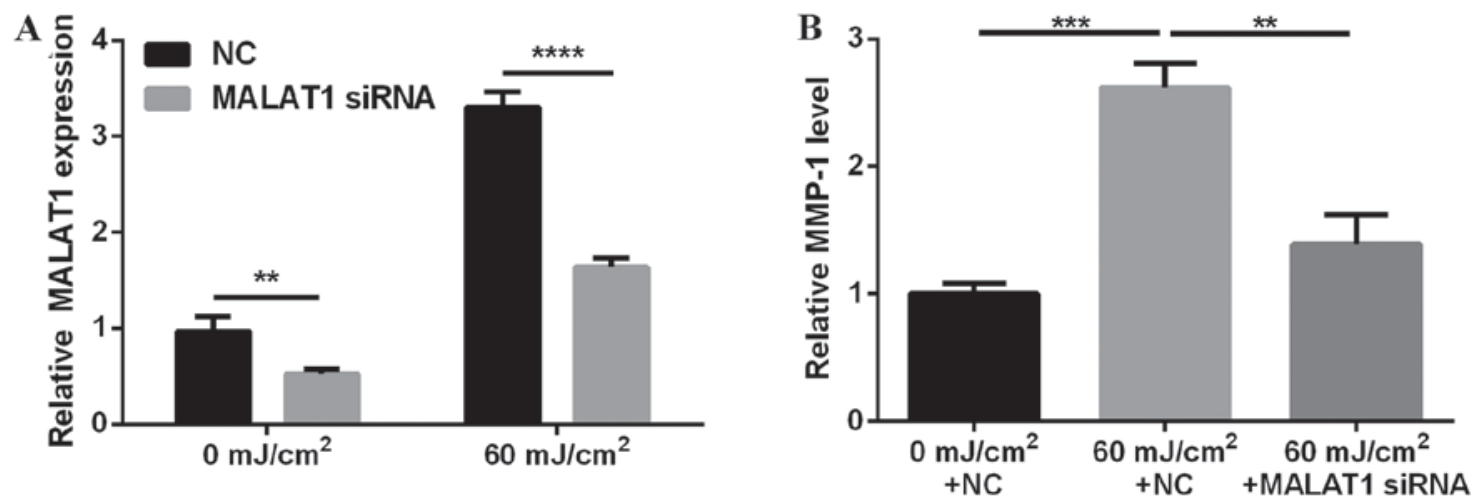

Figure 2. MALAT1 siRNA inhibits UVB-induced MMP-1 secretion. (A) Effect of MALAT1 siRNA on MALAT1 expression in fibroblasts, detected by reverse transcription-quantitative polymerase chain reaction. (B) Effect on MMP-1 expression levels following fibroblast irradiation with $60 \mathrm{~mJ} / \mathrm{cm}^{2} \mathrm{UVB}$ and MALAT1 siRNA, detected by enzyme-linked immunosorbent assay. ${ }^{* * * * *} \mathrm{P}<0.0001$ and ${ }^{* *} \mathrm{P}<0.01 \mathrm{vs.} \mathrm{NC.}{ }^{* * * *} \mathrm{P}<0.001 \mathrm{vs} .0$ mJ $/ \mathrm{km}^{2}+\mathrm{NC}$. MALAT1, metastasis-associated lung adenocarcinoma transcript 1; siRNA, small interfering; UVB, ultraviolet B; MMP-1, matrix metalloproteinase-1; NC, negative control.

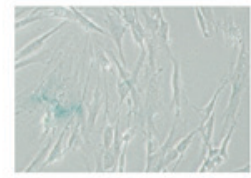

$$
0 \mathrm{~mJ} / \mathrm{cm}^{2}
$$
$+\mathrm{NC}$

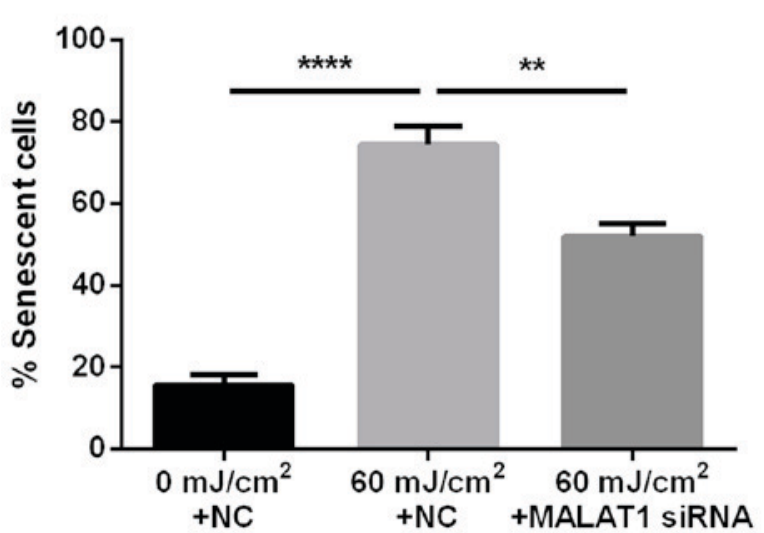

Figure 3. MALAT1 siRNA suppresses UVB-induced fibroblast senescence. Ratio of senescent cells detected via a $\beta$-galactosidase SA- $\beta$-Gal kit, following $60 \mathrm{~mJ} / \mathrm{cm}^{2} \mathrm{UVB}$ irradiation and then intervention with MALAT1 siRNA. Magnification, $\mathrm{x} 40 .{ }^{* * * * *} \mathrm{P}<0.0001 \mathrm{vs} .0 \mathrm{~mJ} / \mathrm{km}^{2}+\mathrm{NC} ;{ }^{* * *} \mathrm{P}<0.01 \mathrm{vs} .60 \mathrm{~mJ} / \mathrm{km}^{2}$ + NC. MALAT1, metastasis-associated lung adenocarcinoma transcript 1 ; siRNA, small interfering RNA; UVB, ultraviolet B; NC, negative control.

with MALAT1 siRNA (52.1\%) compared with the UVB irradiation group $(\mathrm{P}<0.01$ and $\mathrm{P}<0.0001 ;$ Fig. 3$)$.

MALAT1 siRNA inhibits UVB-induced ERK phosphorylation in fibroblasts. Activation of MAPK signaling is important in photo-aging. The present study investigated the effect of the silencing of MALAT1 on activation of MAPK signaling pathway elements, induced by UVB. The results demonstrated that $60 \mathrm{~mJ} / \mathrm{cm}^{2} \mathrm{UVB}$ upregulated ERK, JNK and p38 phosphorylation levels, however MALAT1 siRNA inhibited UVB-induced ERK phosphorylation $(\mathrm{P}<0.01)$, with no significant influence on JNK and p38 phosphorylation levels (P>0.05; Fig. 4).

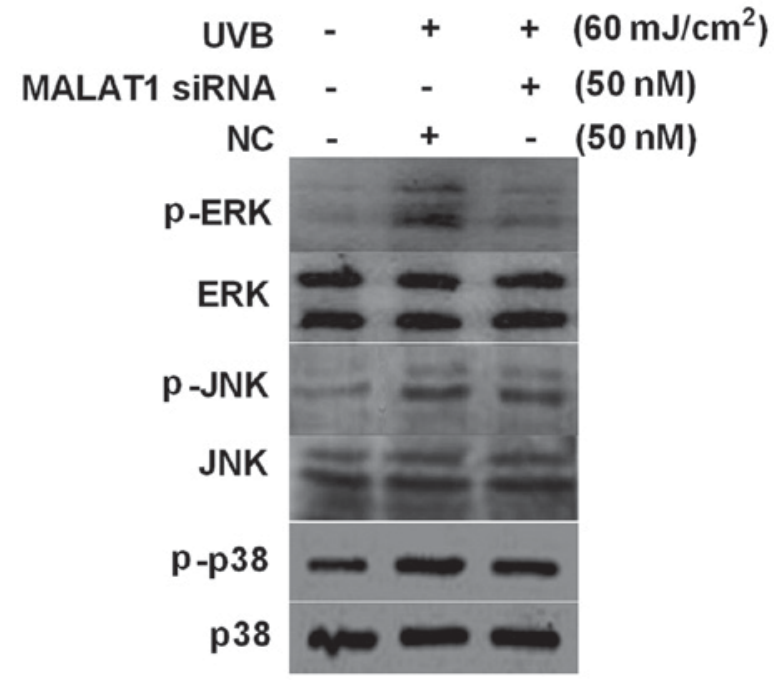

Figure 4. MALAT1 siRNA suppresses UVB-induced p-ERK activation in fibroblasts. Effect of $60 \mathrm{~mJ} / \mathrm{cm}^{2}$ UVB irradiation and MALAT1 siRNA on fibroblast p-ERK, p-p38 and p-JNK expression levels, detected by western blot analysis. MALAT1, metastasis-associated lung adenocarcinoma transcript 1; siRNA, small interfering; UVB, ultraviolet B; NC, negative control; p, phosphorylated; ERK, extracellular signal-regulated kinase, JNK, c-Jun N-terminal kinase.

UVB-induced MALAT1 expression is independent of ROS generation. ROS alters the expression levels of numerous molecules. The present study examined whether ROS underlies UVB-induced MALAT1 alteration using NAC, which is a ROS scavenger. The effects on MALAT1 expression in NAC-pretreated cells were investigated. The results indicated an increase in ROS content following fibroblast irradiation with UVB, which was then inhibited by NAC. However, NAC did not have a significant effect on MALAT1 expression $(\mathrm{P}=0.2307)$. These findings indicated that UVB-induced MALAT1 expression is not dependent on ROS generation $(\mathrm{P}<0.01, \mathrm{P}<0.001$ and $\mathrm{P}<0.0001$ respectively; Fig. 5).

\section{Discussion}

It has recently been demonstrated that microRNA expression profiles within non-coding RNA alter in fibroblasts following 
A

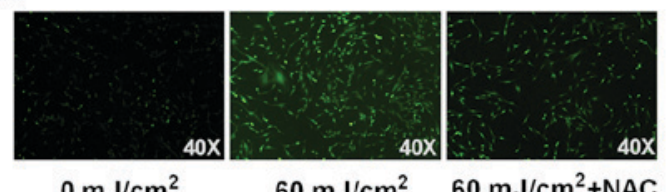

$0 \mathrm{~mJ} / \mathrm{cm}^{2}$

$60 \mathrm{~mJ} / \mathrm{cm}^{2} \quad 60 \mathrm{~mJ} / \mathrm{cm}^{2}+\mathrm{NAC}$

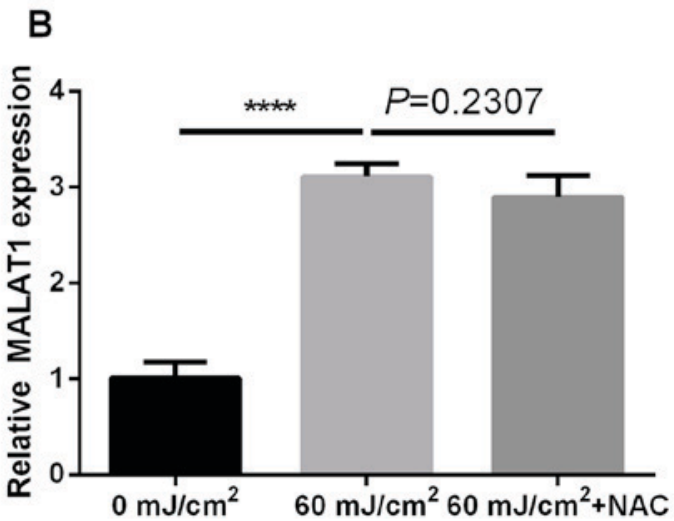

Figure 5. UVB-induced MALAT1 is independent of ROS generation. Effect on (A) ROS generation (magnification, x40) and (B) MALAT1 expression following fibroblast irradiation with $60-\mathrm{mJ} / \mathrm{cm}^{2} \mathrm{UVB}$, and then addition of NAC, detected by flow cytometry and reverse transcription-quantitative polymerase chain reaction respectively. ${ }^{* * * * *} \mathrm{P}<0.0001$ and ${ }^{* * * *} \mathrm{P}<0.001 \mathrm{vs} .0 \mathrm{~mJ} / \mathrm{km}^{2} ;{ }^{* *} \mathrm{P}<0.01 \mathrm{vs} .60 \mathrm{~mJ} / \mathrm{km}^{2}+\mathrm{NAC}$. MALAT1, metastasis-associated lung adenocarcinoma transcript 1; UVB, ultraviolet; ROS, reactive oxygen species; NAC, N-acetyl-L-cysteine.

administration of UV irradiation (15). An additional study verified that two microRNAs, miR-146a and miR-155, potentially regulate photo-aging via targeting $S m a d 4$ and $c$-Jun regulation $(16,17)$. The role of lncRNAs in UV-induced photo-aging of skin remains to be elucidated.

The potential of UV radiation to alter lncRNA expression profiles within skin cells has implications on numerous important biological functions associated with lncRNAs. Hall et al (18) demonstrated that UVB-induced lincRNA-p21 is important in keratinocyte apoptosis. The authors previously demonstrated that >two-fold alterations in the expression of 807 lncRNAs occurred, following melanocyte irradiation with UVB (14), including a markedly increased expression of MALAT1 (data not shown). MALAT1, a well-known cancer-promoting gene within lncRNAs, regulates numerous biological activities including tumor proliferation, metastasis and epithelial-mesenchymal transitioning (19). The current study revealed increases in MALAT1 expression following UVB irradiation of fibroblasts, indicating a potentially important role of MALAT1 in the photo-aging of fibroblasts.

MMP-1 is an important indicator of photo-aging within fibroblasts. In the present study, MMP-1 content increased within fibroblasts following $60 \mathrm{~mJ} / \mathrm{cm}^{2}$ UVB irradiation, suggesting aging of fibroblasts. MMP-1 secretory volume was markedly reduced in photo-aged fibroblasts following silencing of MALAT1 expression, indicating MALAT1 1 promoted photo-aging of fibroblasts. Cell senescence staining experiments confirmed the ability of MALAT1 siRNA to suppress UVB-induced photo-aging of fibroblasts.

The MAPK signaling pathway is activated during photo-aging of fibroblasts (4). Furthermore, recent studies have demonstrated an interaction between MALAT1 and the MAPK signaling pathway $(10,12)$. Therefore, the present study explored the effects of MALAT1 on MAPK signaling in fibroblasts. The results demonstrated MALAT1 siRNA inhibited UVB-induced p-ERK activation, indicating MALAT1 may participate in the photo-aging of fibroblasts via activation of the ERK/MAPK signaling pathway elements.

The authors previously demonstrated that UVB-induced lncRNAs (including lnc-CD1D-2:1) depend on ROS generation (14), and therefore, further proceeded to investigate whether UVB-induced MALAT1 upregulation is ROS-dependent. The results demonstrated no inhibition of MALAT1 expression by NAC (a ROS scavenger), indicating UVB-induced MALAT1 upregulation is independent of ROS. In conclusion, MALAT1 may participate in UVB-induced photo-aging via regulation of the ERK/MAPK signaling pathway elements and UVB-induced MALAT1 upregulation does not depend on ROS generation.

\section{Acknowledgements}

The present study was supported by the Science and Technology Plan of Hunan province (grant no. 2013SK3056) and the New Xiangya Talent Projects of the Third Xiangya Hospital of Central South University (grant no. JY201623).

\section{References}

1. Ham SA, Kang ES, Lee H, Hwang JS, Yoo T, Paek KS, Park C, Kim JH, Lim DS and Seo HG: PPARס inhibits UVB-induced secretion of MMP-1 through MKP-7-mediated suppression of JNK signaling. J Invest Dermatol 133: 2593-2600, 2013.

2. Stoebner PE and Meunier L: Photoaging of face. Ann Dermatol Venereol 135: 1S21-1S26, 2008 (In French).

3. Park G, Baek S, Kim JE, Lim TG, Lee CC, Yang H, Kang YG, Park JS, Augustin M, Mrosek M, et al: Flt3 is a target of coumestrol in protecting against UVB-induced skin photoaging. Biochem Pharmacol 98: 473-483, 2015.

4. Kim MJ, Woo SW, Kim MS, Park JE and Hwang JK: Anti-photoaging effect of aaptamine in UVB-irradiated human dermal fibroblasts and epidermal keratinocytes. J Asian Nat Prod Res 16: 1139-1147, 2014.

5. He X, Bao W, Li X, Chen Z, Che Q, Wang H and Wan XP: The long non-coding RNA HOTAIR is upregulated in endometrial carcinoma and correlates with poor prognosis. Int J Mol Med 33: 325-332, 2014. 
6. Li Y and Wang X: Role of long noncoding RNAs in malignant disease (Review). Mol Med Rep 13: 1463-1469, 2016.

7. Xu S, Sui S, Zhang J, Bai N, Shi Q, Zhang G, Gao S, You Z, Zhan C, Liu F and Pang D: Downregulation of long noncoding RNA MALAT1 induces epithelial-to-mesenchymal transition via the PI3K-AKT pathway in breast cancer. Int J Clin Exp Pathol 8: 4881-4891, 2015.

8. Dey BK, Mueller AC and Dutta A: Long non-coding RNAs as emerging regulators of differentiation, development, and disease. Transcription 5: e944014, 2014.

9. Uchida S and Dimmeler S: Long noncoding RNAs in cardiovascular diseases. Circ Res 116: 737-750, 2015.

10. Liu JY, Yao J, Li XM, Song YC, Wang XQ, Li YJ, Yan B and Jiang Q: Pathogenic role of lncRNA-MALAT1 in endothelial cell dysfunction in diabetes mellitus. Cell Death Dis 5: e1506, 2014.

11. Wei Y and Niu B: Role of MALAT1 as a prognostic factor for survival in various cancers: A systematic review of the literature with meta-analysis. Dis Markers 2015: 164635, 2015.

12. Wu XS, Wang XA, Wu WG, Hu YP, Li ML, Ding Q, Weng H, Shu YJ, Liu TY, Jiang L, et al: MALAT1 promotes the proliferation and metastasis of gallbladder cancer cells by activating the ERK/MAPK pathway. Cancer Biol Ther 15: 806-814, 2014.

13. Livak KJ and Schmittgen TD: Analysis of relative gene expression data using real-time quantitative PCR and the 2(-Delta Delta C(T)) method. Methods 25: 402-408, 2001.
14. Zeng Q, Wang Q, Chen X, Xia K, Tang J, Zhou X, Cheng Y, Chen Y,Huang L, Xiang H, et al: Analysis of lncRNAs expression in UVB-induced stress responses of melanocytes. J Dermatol Sci 81: 53-60, 2016.

15. Syed DN, Khan MI, Shabbir M and Mukhtar H: MicroRNAs in skin response to UV radiation. Curr Drug Targets 14: 1128-1134, 2013.

16. Li W, Zhou BR, Hua LJ, Guo Z and Luo D: Differential miRNA profile on photoaged primary human fibroblasts irradiated with ultraviolet A. Tumour Biol 34: 3491-3500, 2013.

17. Song J, Liu P, Yang Z, Li L, Su H, Lu N and Peng Z: MiR-155 negatively regulates c-Jun expression at the post-transcriptional level in human dermal fibroblasts in vitro: Implications in UVA irradiation-induced photoaging. Cell Physiol Biochem 29: 331-340, 2012.

18. Hall JR, Messenger ZJ, Tam HW, Phillips SL, Recio L and Smart RC: Long noncoding RNA lincRNA-p21 is the major mediator of UVB-induced and p53-dependent apoptosis in keratinocytes. Cell Death Dis 6: e1700, 2015.

19. Gutschner T, Hammerle M and Diederichs S: MALAT1-a paradigm for long noncoding RNA function in cancer. J Mol Med (Berl) 91: 791-801, 2013. 